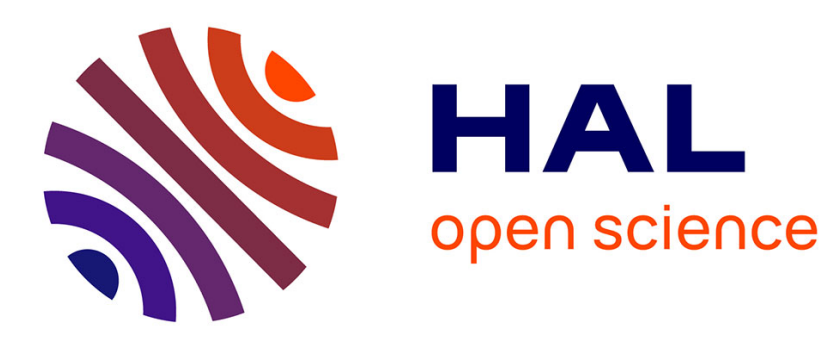

\title{
La peinture dans les recueils collectifs de pièces en vers (1597-1627): un tableau d'images
}

\author{
Véronique V. Adam
}

\section{To cite this version:}

Véronique V. Adam. La peinture dans les recueils collectifs de pièces en vers (1597-1627) : un tableau d'images. Dix-septième siècle, 2009, 245 (4), pp.621-641. hal-00952472

HAL Id: hal-00952472

https://hal-univ-tlse2.archives-ouvertes.fr/hal-00952472

Submitted on 28 Feb 2014

HAL is a multi-disciplinary open access archive for the deposit and dissemination of scientific research documents, whether they are published or not. The documents may come from teaching and research institutions in France or abroad, or from public or private research centers.
L'archive ouverte pluridisciplinaire HAL, est destinée au dépôt et à la diffusion de documents scientifiques de niveau recherche, publiés ou non, émanant des établissements d'enseignement et de recherche français ou étrangers, des laboratoires publics ou privés. 


\section{La peinture dans les recueils collectifs de pièces en vers (1597-1627) : un tableau d'images}

Véronique Adam

(Université Toulouse II-Le Mirail, CRI-Grenoble)

Les recueils collectifs de pièces en vers des années 1597 à $1627^{1}$ diffusent souvent pour la première fois les textes d'auteurs éditées ensuite en œuvre complète. Elles témoignent ainsi des poètes montants - Malherbe prend peu à peu la première place dans les anthologies ${ }^{2}$ — et se placent sous l'égide de la nouveauté dans leurs préfaces, témoins privilégiés des tentatives de cette poésie Louis XIII, saturée de clichés et d'images déjà connues, de se montrer plus originale qu'il n'y parait. Les poèmes liés à la peinture pourraient être le symbole, modèle et contre-modèle, de cette ambiguïté des anthologies et de cette poésie : ils y sont rares, en cela originaux. Ils croisent aussi idéalement les écueils de la poésie de ce début de siècle puisqu'ils sont eux aussi inscrits dans une topique rebattue, lieux de fiction amoureuse, relevant d'une esthétique attendue, illusions parfaites de la nature plaçant en leur cœur, la question de l'imitation. La poésie de ce premier XVII ${ }^{\mathrm{e}}$ siècle trouverait donc un double aussi imparfait qu'elle, propice au renouvellement ses figures : la syllepse, hésitant entre le littéral et le figuré, fréquemment utilisée pour redonner vie au cliché, semble trouver dans le tableau un symbole parfait. Il joint l'objet tangible à l'art qui le transforme en peinture, le visage d'un artiste également image du poète. En prenant pour sujet et objet le tableau, la poésie part en quête de nouvelles figures rhétoriques ou mythologiques. Au lieu de l'imitation, elle trouve l'invention. Au lieu de l'analogie, la dissémination de l'image. Après un examen de la présence du thème pictural dans les anthologies, nous verrons comment la poésie redonne corps et force imaginaire aux topiques de la représentation picturale. Présence tangible et fantasmatique qu'une étude sur la matérialité du tableau viendra prolonger. Enfin, le peintre, inscrit dans une fiction mythologique, montrera un dernier étagement de cette volonté qu'à la poésie d'entraîner la peinture vers un renouvellement de l'univers poétique.

\footnotetext{
${ }^{1}$ On trouvera à la fin de cet article la liste des anthologies consultées, classées par éditeurs (notamment R. Petit-Val, M. Guillemot, T. De Bray). Nous n'avons retenu que les anthologies généralistes et écarté les anthologies thématiques ou de circonstances ( telles les Muses en deuil, Paris, Du Bray, 1620) ou les anthologies ne proposant qu'un genre unique (satiriques par exemple), puisqu'à de rares exceptions, que nous signalerons, elles ne répondaient pas à notre enquête. Nous avons donc privilégié les anthologies sans unité apparente et celles qui témoignaient d'un certain succès sensibles dans leurs multiples rééditions. Voir aussi F. Lachèvre, Bibliographie des recueils collectifs de poésies publiés de 1597 à 1700, Paris, Leclerc, 1903-1905, 4 t.

${ }^{2}$ Malherbe, dans les éditions de Du Bray, passe à la première place en 1627, remplaçant Du Perron. Les auteurs importants sont soit placés parmi les premiers ou en dernière position ( tel D’Urfé en 1609 chez Du Bray ou Malherbe chez Daré en 1626). La présence dans un recueil en premier lieu peut aussi être simplement due au fait que l'auteur vient de mourir ( tel Vermeil en 1600 chez Guillemot).
} 
- Etat des lieux : une stratégie de la rareté

Les emprunts à l'univers pictural recouvrent un clivage éditorial : on ne trouve aucune ekphrasis de tableau chez Du val, Guillemot ou Corrozet, seuls trois poèmes évoquant un peintre ou sa pratique en général sont recueillis. La peinture est limitée à un vocable courant dont on ne peut du reste assurer qu'il relève de son seul domaine (portrait ou trait par exemple). Les Marguerites d'Aubert, sorte de dictionnaire thématique comportant plusieurs centaines d'entrées thématiques, n'offrent que deux entrées sur la peinture ( « un peintre s'égayant de son tableau » et « une laide peinture »). Elles ignorent le thème lorsqu'elles abordent la poésie et la lettre $P$. On propose une entrée «poésie et musique ». En revanche, les vers sur des portraits peints se voient offrir une part croissante chez Toussaint Du Bray, l'éditeur qui va éclipser Du Val et Guillemot : une quinzaine de pièces dans les volumes de 1609, 1620 et 1627, d'auteurs considérés comme majeurs ou montants, avec une part croissante d'œuvres entièrement consacrées à un peintre ou à un tableau, plutôt que seulement des vers convoquant ponctuellement le mot « peinture » ou « portrait». Il faut attendre l'anthologie de Sorel en 1647 pour voir présente une métaphore picturale dès la préface et qu'on la lie au mode même de composition de l'anthologie :

Avant que de faire des bouquets, l'on a des amas de fleurs préparés; et le peintre a diverses couleurs sur sa palette avant que de commencer un portrait ${ }^{3}$

Mais cette anthologie n'est pas en vers quoiqu'elle reprenne le motif de la fleur composant le bouquet, image traditionnelle des anthologies que nous étudions.

Cette rareté globale de la peinture peut avoir plusieurs causes : une concurrence externe puisque ne sont publiés en anthologie, la plupart du temps, que les textes qu'on ne trouve pas ailleurs. Or des galeries de tableaux sont éditées, sous l'influence de Marino et de sa Galeria (1620) qu'on retrouve imitées dans des œuvres complètes. Une concurrence interne est aussi notable : l'idée mimétique est déportée sur le miroir, le songe voire sur la sculpture, plutôt que sur la peinture. On nuancera plus bas ces causes internes. Du reste, dans l'anthologie, la rareté est justement une stratégie constante : recherchée par les auteurs de préface qui veulent des textes inconnus, sensibles dans l'édition limitée de vers d'auteurs reconnus (au contraire des auteurs clairement qualifiés de secondaires et pourtant mieux représentés en nombre de vers), les poèmes-peintures fonctionnent donc comme des apax mais ils ont entre eux une certaine cohérence. Ainsi, Boisrobert peut se montrer exemplaire de l'intérêt non négligeable pour l'univers pictural dans l'anthologie et du cadre global dans lequel elle est inscrite : nouveau venu dans l'anthologie de 1627 de Du Bray, il propose plusieurs pièces liant

\footnotetext{
${ }^{3}$ Ch. Sorel, «Préface », Nouveau Recueil des pièces les plus agréables de ce temps, Paris, Sercy, 1644. Il n’y recueille que des textes de prose poétique.
} 
poésie et peinture. Parallèlement, ses pièces passent essentiellement par des « rites d'autorisation ${ }^{4}$ » pour justifier sa présence dans l'anthologie, soit en optant pour des genres institués (ode, sonnet) soit en convoquant des autorités littéraires et politiques. Il légitime ainsi sa présence, en rédigeant des vers en l'honneur de Malherbe et Richelieu, eux-mêmes auteurs publiés dans le recueil. A l'intérieur de ses évocations picturales, il reprend justement le thème imposé par une autre autorité, Honoré d'Urfé. Ce dernier publiait dans le recueil précédent ${ }^{5}$, plusieurs sonnets sur des portraits dont il louait le charme et l'effet, montrant que la vérité du tableau importait moins que la vérité de son effet, nous y reviendrons. D’Urfé après le succès de l'Astrée disparaît des anthologies puisque ses œuvres sont publiées séparément. Boisrobert le remplace et propose donc des pièces sur des portraits dans le cadre de cette légitimation par les autorités. A l'intérieur de ses vers, il reprend comme modèles de peintre les deux figures reconnues dans d'autres extraits de recueils collectifs d'anthologie évoquant la peinture: Apelle déjà là dans les vers de Ronsard cités dans les Marguerites $^{6}$ et Michel Ange convoqué dès $1620^{7}$. Ils sont préférés à tous les autres peintres. Boisrobert ignore l'allusion à la mouche de Giotto et aux oiseaux de Zeuxis, présentes ailleurs. Il n'évoque qu'un peintre contemporain, Lartigue, auteur d'une fresque dans l'église de Corme Royal, inconnu dans les autres volumes, et assez surprenant: les peintres contemporains des autres anthologies sont généralement convoqués à cause de leur double autorité sociale (peintre du roi ou de la cour) et esthétique, tels Arley, Freminet ou Janet. Boisrobert montre ainsi que les poèmes picturaux s'inscrivent dans une nouveauté contrôlée, autorisée mais soumise à de multiples formes d'imitatio: imitatio d'une autorité, interne aux anthologies et double (modèles de peintres et de poètes) ; ekphrasis mimétique d'une œuvre d'art par les vers qui la décrivent ; mimesis par le dessin de la nature. Ils deviennent clairement le lieu d'un paradoxe: dans cette mise en abyme des imitations entre elles, le poète, tout en se soumettant à ses modèles, brille et modifie la topique qu'on lui avait assignée.

Fort logiquement, si cette limitation de la référence picturale, dans sa présence et son univers de référence, se retrouve dans la topique qu'on lui assigne, la poésie tente d'aller au-delà des ces frontières rhétoriques et esthétiques : ainsi la peinture est une poésie muette, et la poésie, une

\footnotetext{
${ }^{4}$ J'emprunte cette expression à Franck Lestringant, André Thevet Cosmographe Des Derniers Valois, Genève, Droz, 1991, p.265. Il désigne un moment d'échange symbolique entre un auteur reconnu et un auteur qui veut s'y référer comme à une autorité, tel Scève prétendant avoir découvert le tombeau de la Laure de Pétrarque. Plus généralement, les rites d'autorisation désigne ici pour moi le recours explicite par un poète à de multiples éléments relevant d'une autorité (genre, discours, pensée) afin de légitimer sa présence au côté de cette auctoritas, recours initiatique d'intronisation qui précède l'édition de textes plus personnels du poète.

5 Baudoin, qui édite pour Du Bray en 1620 le Second livre des Délices de la poésie française, profite du succès de l'auteur de l'Astrée pour publier ses vers.

${ }^{6}$ Cité dans les Marguerites poétiques, Lyon, 1613, par Ronsard, et dans le Second livre des Délices, éd. Baudoin pour Du Bray, 1620, par d’Urfé, p. 41 et Picardière, p. 756 ; éd Du Bray, 1627, par Boisrobert, p. 482.

${ }^{7}$ Maynard, p. 1045, Du Bray, 1621, et Maynard, Boisrobert, Du Bray, 1627, p. 374, p. 482.
} 
peinture parlante; toutes deux sont placées en regard de la nature, souvent supérieure. La poésie modifie ces deux topoï.

- La réécriture de la topique picturale.

Les recueils collectifs nuancent cette topique de la chose muette : les vers, au lieu d'exprimer la passion et les sentiments muets du tableau, traduisent le silence pictural, sans faire parler les personnages du tableau et en légitimant l'absence d'expression. La poésie refuse de stigmatiser le défaut de son double privé de discours. Elle montre la réussite de l'effet du tableau silencieux dans cette pièce de Malherbe rééditée trois fois :

L'art aussi bien que la nature

Eût fait plaindre cette peinture,

Mais il a voulu figurer

Qu'aux tourments dont la cause est belle,

La gloire d'une âme fidèle

Est de souffrir sans murmurer ${ }^{8}$.

Le silence reflète la vertu de la sainte et montre la réussite de cette image pieuse, moins soumise à l'artifice du silence pictural qu'à la nature conjointe de la douleur et du tableau muets. Cette douleur inaudible justifie le silence pictural et va de pair avec l'anecdote de Pline sur Timanthe, justement reprise dans deux anthologies : le peintre Timanthe incapable de rendre compte de la douleur d'un père,

Ne pouvant exprimer des traits de son pinceau

Le deuil de ce grand Roi lui voila le visage ${ }^{9}$

Malherbe n'a fait qu'opérer un transfert synesthésique de la douleur invisible à la douleur inaudible. Le topos de la chose muette est renouvelé deux fois, par sa légitimation mimétique et par la réécriture du modèle antique (Pline) et moderne (Du Perron).

Le silence pictural tourne tout de même à l'avantage du poète qui y découvre la possibilité d'une figure antithétique et choisit de transférer non plus un sens vers un autre mais une sensation vers une autre. A l'exact opposé de la douleur cachée, silencieuse et inaudible surgit le plaisir; le silence du tableau devient non plus extase mystique, mais fantasme érotique. On inverse les rôles : la femme, dans la réalité indifférente, montre dans sa peinture, en se taisant, le signe d'un consentement attendu :

Portrait de mes larmes baigné $[\ldots]$

\footnotetext{
${ }^{8}$ Malherbe, « sur l'Image d'une Sainte Catherine », les Délices, déd. Pr. De Conti, Du Bray, 1620, p. 280 et Second livre, éd. Baudoin/Du Bray, 1620 p. 165. Cette pièce est répertoriée en 1627 dans l'index, mais absente du volume. Par commodité, puisque deux éditions d'anthologie sont diffusées par Du Bray en 1620, on citera d'un côté l'édition des Délices avec la mention de la dédicataire, la Princesse de Conti (Conti/Du Bray), et l'édition des Second Délices, par la mention de son éditeur scientifique, Jean Baudoin (Baudoin/Du Bray).

${ }^{9}$ Du Perron, « Confession amoureuse », Conti/Du Bray, 1620, p.117.
} 
Portrait de mes feux animés [...]

Tu me réponds si doucement

Avecque ton muet silence

Qu'il me semble un consentement ${ }^{10}$.

Le silence condense les sensations même antinomiques. Ce balancement entre la vertu du silence pictural et son plaisir lui confère naturellement un sens moral, bientôt vice monstrueux chez la femme inconstante. Le poète, à l'instar de Timanthe, reprend à son tour l'artifice du peintre en refusant lui aussi de dire et montrer ce vice innommable

En me taisant aussi je voile tes forfaits

$\mathrm{Au}$ lieu de mes discours il faut voir tes effets,

Ce qu'on peut essayer n'a besoin de langage.

Les limites de l'expression langagière de la peinture et de la poésie sont ainsi motivées et tentent au final de réduire la distance entre le modèle et son dessin en jouant d'un paradoxe : le silence est une preuve parlante de la nature de la réalité et du parfait mimétisme des arts. La réflexion sur l'écart entre la nature, en particulier humaine, et sa représentation prise dans ce jeu de renversement et d'inversion, offre au poète une opportunité : continuer ce paradoxe en complétant l'antithèse du silence et du langage, par une autre, opposant la peinture vive de la nature et celle figée et morte de l'artiste. L'expression «vive peinture » est bien plus récurrente dans les anthologies que la peinture littérale, et rime alors avec nature ${ }^{11}$. " vive » suggère toujours la présence conjointe d'un mouvement et d'un souffle vital dans les éléments naturels ${ }^{12}$. Le tableau face à cette peinture vive, fort logiquement, est le lieu de la présence d'objets morts. Mais le peintre s'y montre capable de les animer :

[ces beaux yeux] Bien qu'ils soient morts en ce portrait

Ils ont tant de grâce et d'attrait,

Leur flamme est si vive et si claire,

Qu'on brûle aussi tôt à les voir :

Eux-mêmes que peuvent-ils faire

Si leur portrait à ce pouvoir ${ }^{13}$ ?

\footnotetext{
${ }^{10}$ Motin, « Sur un Portrait », Du Bray, 1609, p. 261 ; Conti/Du Bray, 1620, p. 809.

${ }^{11}$ Maynard, « Ode à M. Racan », Du Bray, 1627, p. 286.

${ }^{12}$ Le vocabulaire pictural reste très banal (teint, couleur, trait, tableau). Le mot «trait » est récurrent car il appartient à l'univers du dessin, un objet mythologique ou guerrier (les traits sont les flèches d'amour lancés par la belle) et une métaphore de la vue encore perçue comme littérale (on croit encore chez les poètes que pour voir l'objet l'œil lance des rayons sur lui qui viennent ensuite se refléter dans l'œil). L'anthologie satirique, qui n'est pas notre objet ici, est la seule, d'un point de vue linguistique, à proposer une pièce qui utilise des termes précis et techniques empruntés à la peinture, tels « le crayon », « le détrempe « le pinceau », « le fond» dans "Le Portrait de Pamphage », Les Muses gaillardes recueillies des plus beaux esprits de ce temps, Du Brueil, Paris, 1609, p. 206-207.

${ }^{13}$ Motin, « Sur un Portrait », Ibid.
} 
Le surgissement d'une concurrence entre nature et peinture confère à cette dernière une force démiurgique et déporte pour un temps la rivalité de la poésie et de la peinture. Les oppositions entre fiction et vérité des arts, plus communes, sont progressivement décentrées sur l'alternative entre la vie et la mort, et surtout sur l'effet produit sur un corps incarné et réel, qui rend caduque le problème de la vérité. Si Du Bellay, dans l'anthologie thématique de G. Corrozet, opposait l'histoire qui « représente le vrai de chaque action et rapporte d'après le vif », et soulignait les « mille fictions » de la peinture et de la poésie ${ }^{14}$, les poètes voient dans les anthologies ultérieures une nuance. Qualifiée de « chose imaginaire ${ }^{15}$, le tableau n'en provoque pas moins un ravissement semblable à celui de la vraie femme. De même que le portrait des yeux morts crée une flamme vive insufflant à son tour du feu dans le corps de l'amant, ailleurs des yeux qui ne sont qu'une « feinte », dont le trait est luimême «feint», font rimer «plaie» avec «vrai» et ressentir une «blessure certaine ${ }^{16}$. Progressivement, les pièces multiplient les transferts et les confusions : de la nature à la peinture, de la femme à son dessin, de la fausseté à la vérité, et pour finir, de la femme réelle à l'imagination du peintre. Qu'importe l'erreur, puisque l'effet est identique de l'amante à son dessin. De l'aveu des poètes-amants, ce qu'ils se mettent à aimer, c'est «l'imagination d'un homme » et les « charmes [empruntés] à la main d'un auteur ${ }^{17} »$. Ainsi brouillées, les frontières de la fiction picturale ne sont plus évaluées à l'aune de leur correspondance avec leurs sources mais en fonction de la finalité qu'elles atteignent vraiment. La mimesis n'est plus considérée en amont mais en aval du tableau. Le peintre et ses charmes rejoint le poète et ses vers (carmen). Cette inversion du regard sur la mimesis peut prendre une autre forme : la force démiurgique du peintre s'inscrit alors dans un combat qui l'oppose à la nature et la mort, lutte qu'il remporte même s'il en meurt.

Fréminet en vivant à l'envie de nature

Donnait avec tant d'art de l'âme à sa peinture,

Que la nature et l'art n'étaient qu'un en ses traits.

Et tant de pouvoir sa main était pourvue,

Que comme il inspirait une âme en ses portraits,

A ceux qui le voyaient il l'ôtait par sa vue.

La nature et la mort enviant l'avantage

De cet art qui mettait leur puissance en partage [...]

En vain ont conspiré de limiter sa vie.

Car il vit en sa mort puisqu'il se peut vanter

Que nature et la mort l'ont fait mourir d'envie ${ }^{18}$.

\footnotetext{
${ }^{14}$ Du Bellay, Le Parnasse des poètes français, éd. Gilles Corrozet pour M. Guillemot, 1607, p. 38.

${ }^{15}$ Boisrobert, « Sur un Portrait fait à plaisir », Du Bray, 1627, p. 481.

${ }^{16}$ D’Urfé, « Sonnet d'un Portrait », Conti/Du Bray, 1620, p. 306.

${ }^{17}$ Boisrobert, «Sur un Portrait fait à plaisir », Du Bray, 1627, p. 483.

${ }^{18}$ Revol, « Epitaphe de Feu Fréminet, peintre du roi », Conti/Du Bray, 1620, p. 994.
} 
Le mimétisme de la nature dans la peinture est inversé, puisque la nature jalouse le peintre qui l'imitait pourtant. Ce n'est plus l'objet naturel que l'on copie, mais le geste du peintre, création partagée entre l'œil, la main et l'âme. Le corps mort du peintre gagne ce paragone dans lequel la poésie et les autres arts sont remplacés par la nature, et le matérialise puisque sa mort prouve l'envie de sa rivale, comme son tableau animé montrait sa supériorité. Ce transfert sur le corps mortel du poète de sa rivalité avec la nature intègre dans la question de la représentation, le problème du temps ${ }^{19}$.

La question de l'image morte de l'artiste et vivante de la nature est résolue et dépassée par l'intervention de la poésie qui ne se contente pas de mettre en scène la rivalité : en assimilant la vie et la mort de l'image à d'autres supports réflexifs, compromis entre nature et peinture, le poète multiplie les concurrents de la sphère picturale et de la nature tout en s'assignant une tâche constante et un rôle unique : représenter tous les modes de représentation, faire une ekphrasis constante non plus de ce qui est peint mais de la manière et la matière du tableau, montrer ce qui borde le tableau et ce que suggère sa création. La poésie au lieu de décrire le dessin, se concentre sur ses marges.

\section{- La matérialité du tableau}

Parmi ces supports réflexifs, certains comme le miroir naturel, le songe, l'ombre et le souvenir, ont été repérés comme caractéristiques du monde des faux semblants baroques, et on note dans les anthologies leur omniprésence, bien plus que les références à la peinture. Les vers sur le miroir et l'ombre, on le sait, font du monde une syllepse totale. L'eau fait ainsi coïncider la nature liquide du tableau naturel, l'image représentée du tourment amoureux, et les larmes signes tangibles de la douleur de l'amant contemplant le miroir. Cette matérialité, fondée sur cette syllepse de l'eau, signifiante pour ce miroir liquide est justement reprise dans le véritable tableau peint, lui aussi palpable et décrit dans sa présence élémentaire, souvent liquide, et matérielle par la poésie. On confère au tableau, une matière qui croise souvent idéalement en les renouvelants, les attributs réflexifs et naturels de l'onde, soulignant comme elle, le croisement réussi du support et de son modèle, entre la nature humaine et la peinture naturelle, confondant le geste du poète, du peintre et de la nature. Trois matérialisations du tableau sont notables et montrent cette volonté de retravailler la topique du miroir naturel.

Les substances du tableau sont inscrites d'abord dans un jeu d'échos matériels et visuels avec le miroir naturel. La concurrence entre deux images mimétiques au lieu de répéter la rivalité de la nature et de la peinture, introduit une contamination du tableau par l'univers imaginaire du miroir

\footnotetext{
${ }^{19}$ Nous retrouverons plus bas cette question du temps lorsque Théophile s'en prend au topos de l'immortalité donnée par les vers et la toile à leur sujet.
} 
liquide. Ce dernier, la plupart du temps lié au mythe de Narcisse, son « ombre », « ses larmes », voit ainsi ses motifs repris dans la contemplation du tableau par l'amant :

Portrait de mes larmes baigné,

Quand de ces beautés éloigné,

Je te dis ma triste aventure,

Que de bien ton ombre me fait [...]

Tu n'es fait que d'huile et de bois,

Et ne suis étonné cent fois

De te voir à mes feux durable.

Mais si tu n'en as rien senti

Il faut que le cours favorable

De mes pleurs t'en ait garanti ${ }^{20}$.

La matérialité du tableau est d'autant plus remarquable qu'elle met l'accent sur des matériaux présents dans leur sens littéral, plus fréquemment utilisés ailleurs pour convoquer une fonction symbolique (le bois de la croix, l'huile de Psyché ou de la lampe qui illumine). Si l'huile des couleurs et le bois du tableau ne se consument pas, ce n'est pas parce que le feu amoureux est figuré. Ce sont les larmes versées, aussi littérales que celles de Narcisse, qui préservent le tableau de la combustion. Mais loin de brouiller l'image reflétée comme dans le mythe, elles la conservent. La syllepse vient renouveler le cliché du feu amoureux et fait surgir le tableau dans un jeu de coïncidence des contraires liant l'ombre à la lumière, le feu à l'eau. On dépasse clairement l'analogie entre les supports réflexifs pour marquer des distinctions et souligner le paradoxe de la matière liquide rendant éphémère ou pérenne une image. C'est avant tout la pointe du poète que l'on perçoit. Ce renouvellement rhétorique du cliché poétique par la matière et l'entrecroisement de deux supports réflexifs, miroir liquide et image peinte, touchent d'ailleurs la poésie elle-même et proposent une seconde forme de matérialisation destinée à distinguer plus avant les modes de représentations. Porchères ${ }^{21}$ invente une matière commune à la poésie et à la peinture littérale et figurée, issue du même élément naturel. Au lieu de l'eau ou l'huile, il offre la cire au peintre et le miel au poète : la poésie n'est pas moins matérielle que la peinture ou le miroir de l'onde :

Avec tous les corps et les âmes,

Nous peignons de traits empruntés

De la beauté de tant de dames

La dame de tant de beautés.

Et comme les sages avettes

Dedans un verger odorant,

\footnotetext{
${ }^{20}$ Motin, « Sur un Portrait », Conti/Du Bray, 1620, p.809.

${ }^{21}$ Porchères, «Stances sur un portait de cire », Le Séjour des Muses ou la crème des bons vers, Rouen, Daré, 1626, p. 380-382.
} 
Vont sur mille et mille fleurettes

La cire et le miel picorant. [...]

Nous cueillons ainsi qu'elles font,

Du suc que l'un et l'autre tire,

De ces vivantes fleurs du Ciel,

$\mathrm{Tu}$ fais la peinture de cire,

Je fais les paroles de miel.

Ce miel figure en mon ouvrage

L'œuvre assez doucement écrit

La douceur de son beau visage,

Et celle de son bel esprit.

D'un peu de cire en ce volume

Tu fais un grand flambeau d'amour,

Lequel jamais ne se consume

Encore qu'il brûle toujours

La métaphore de l'abeille, figure du poète depuis 1'Ion de Platon ${ }^{22}$, est prolongée par des métonymies qui rendent aussi bien compte de la méthode du peintre et du poète (l'abeille rassemble du pollen pour créer le miel et la cire, de même le poète et le peintre empruntent à plusieurs beautés des traits pour faire une seule beauté) que de leurs instruments (douceur et éclat). Dans la méthode, on retrouve une définition constante de la peinture dans les anthologies : la beauté est un assemblage d'éléments distincts au départ, choisis chacun pour leur beauté. Boisrobert voit dans la peinture des « assemblages de fards » ${ }^{23}$; Picardière montre que le Ciel pour faire un portrait « assemble [...] ce qu'il y a de plus beau [et] s'y portrait comme dans un tableau $»^{24}$. On retrouve une idée développée à l'identique chez les théoriciens de 1 ' $\operatorname{art}^{25}$. Le travail de synthèse artistique métaphorisée par la synthèse matérielle du miel et de la cire (présente aussi dans les fards), permet clairement de dépasser la simple analogie avec la nature, puisque la matière artistique dépasse la matière naturelle dans un nouveau jeu de syllepse : la cire ne fond pas. D’une manière générale, la poésie utilise la peinture pour dédoubler les métaphores et les analogies stéréotypées entre des éléments figurés et littéraux, en les partageant avec d'autres techniques elles-mêmes analogiques, compensant ce faisant

\footnotetext{
${ }^{22}$ Voir J.H. Waszieck, Biene und Honig als Symbol des Dichters und der Dichtung in der griechisch-römischen Antike [Abeilles et miel comme symbole du poète et de la poésie dans l'antiquité gréco-romaine], Opladen, Westdeuscher Verlag, 1974. On retrouve notamment au début du XVII siècle le vers « pilloter », pour désigner conjointement le mouvement de l'abeille et les multiples choix d'éléments épars que le poète réunit dans son œuvre.

${ }^{23}$ Boisrobert, «Sur un portrait fait à plaisir », Du Bray, 1627, p. 381. Le fard désigne le pigment ou la cire, qui sert de base au maquillage ou à la couleur, il a aussi une nuance de fausseté. Cette métaphore est fréquente dans la théorie artistique. Victor Stoichita lui consacrera en 2009 à Fribourg un séminaire. Voir aussi Catherine Lanoë, La poudre et le fard : une histoire des cosmétiques de la Renaissance aux Lumières, Paris, Champs Vallon, « époques », 2008.

${ }^{24}$ Picardière, « Hymne de la Reine », Conti/Du Bray, p. 931, Baudoin/Du Bray, p. 758.

${ }^{25}$ Roger de Piles, Dialogue sur le coloris, Paris, N. Langlois, 1699, p.7, définit le rôle du peintre comme celui qui « ne doit pas imiter toutes les couleurs qui se présentent indifféremment, il ne doit choisir que celles qui lui conviennent, auxquelles (s'il le juge à propos) il en ajoute d'autres qui puissent produire un effet tel qu'il l'imagine pour la beauté de son ouvrage ».
} 
le défaut rhétorique d'une poésie de seconde main. La gémellité matérielle et technique entre l'abeille, le poète et le peintre, de même que la correspondance entre le miroir naturel et la peinture semblent nous faire glisser d'un monde analogique à un principe plus taxinomique : nous aurions, pour reprendre une distinction aristotélicienne, d'un côté le genre mimétique lié à l'élément liquide, de l'autre des espèces qui en découleraient, le miroir naturel et l'eau, le tableau d'huile et de cire, le poème et son miel. Ces trois supports réflexifs sont similaires dans leur fonctionnement (choix puis synthèse) mais non dans leurs effets (tristesse de l'eau, douceur des vers, éclat de la peinture).

Le troisième et dernière mode de matérialisation du tableau dans les vers consiste à assimiler le sujet du tableau et les éléments matériaux picturaux : tel peintre décide de peindre «l'œil de sa Dame avec un charbon » :

\section{Si pour représenter la cause \\ L'effet ne nous sert pas de peu, \\ Trouvez-vous une étrange chose \\ Que le charbon marque le feu ${ }^{26}$ ?}

La nature du tableau donne à la représentation mimétique une troisième dimension. Elle lie le sujet illustré à la matière picturale et instaure une logique élémentaire entre la manière de peindre, au charbon, et le modèle reproduit, le feu des yeux éclatants de la femme. La syllepse touche donc la matière elle-même, puisque le charbon est utilisé pour figurer le trait du dessin et donner une cause littérale et physique, naturelle, pour tout dire, à l'effet qu'il produit. La correspondance matérielle entre le tableau et son sujet se retrouve dans des pièces intégrant quant à elles moins la réalité physique des éléments qu'une pratique magique et rituelle reliant d'une manière plus générale l'image et son modèle : Malherbe, dans une pièce reprise dans les trois anthologies, évoque l'attentat contre le roi qui a touché toute la France rappelle que «par magie en frappant une image/ celui qu'elle figure en reçoit le dommage ${ }^{27}$. Boisrobert va plus loin et suggère qu'on brûle le tableau de la femme qu'il aime :

Cette belle et parfait image

Fais qu'on la brûle devant toi

Qu'une fois pour l'amour de moi

Je la puisse voir enflammée

Et que mes désirs continus

Retournant, comme ils sont venus

Aillent avec elle en fumée ${ }^{28}$.

\footnotetext{
${ }^{26} \mathrm{Du}$ Brun, «A un qui se moquait de ce qu'il peignait l'œil de sa Dame avec un charbon », Conti/ Du Bray 1620, p. 1134.

${ }^{27}$ Malherbe, «Stances sur ce qui fut attenté contre le Roi le lundi 19 décembre », Du Bray, 1609, p. 269. Pièce rééditée en 1620 , p. 817 et 1627, p. 729 , sans variante.

${ }^{28}$ Boisrobert, « Sur un portrait », Du Bray, 1627, p. 484.
} 
Le tableau reprend certaines des fonctions assignées plus fréquemment ailleurs au songe érotique. Ce dernier contamine à l'instar du miroir, l'organisation du fantasme: le songe permet de rendre tangible la belle indifférente, de la posséder et de passer de la douleur de l'absence à une présence plaisante, dont on souligne toujours le caractère évanescent. De même ici, d'une manière similaire ou inversée, l'objet amoureux inaccessible devient sensible au feu même de l'amant qui lui transfère littéralement son incandescence. Il y trouve la double satisfaction de voir le dessin féminin subir la douleur qu'il ressent, rendue ce faisant évanescente en lieu et place de l'ombre de la femme dans le songe. La nature combustible du tableau permet de reprendre l'organisation et la fonction d'un support réflexif plus fréquent, le songe, mais il a un caractère palpable qui le transforme en substitut du corps féminin. Fétichisation expiatoire qui assure aux vers une originalité rhétorique certaine, allant bien au-delà d'une syllepse.

La matérialité du tableau lui confère une réalité naturelle. Ce réel pousse à l'extrême la vraisemblance. Le bois du tableau, le dessin au charbon ou l'image communiquant avec son modèle et ses effets, montrent l'existence d'une relation essentielle et physique, et non plus seulement visuelle, entre la matière et la mimesis, le sujet et son portrait pictural. Il n'en demeure pas moins vrai que la peinture est en-deçà de la poésie. Celle-ci hésite en effet dans les anthologies entre deux tentations : léguer à l'art une immatérialité que la peinture ne peut appréhender, puisque faite de matériaux tangibles ou décrire, plutôt que le tableau et les supports réflexifs, l'image sensible du cœur de chair, dernier avatar de cette quête de la matière la plus parfaite à rendre compte de ce qu'on veut représenter. La beauté était un assemblage de matières, organisé entre l'œil de la femme et la main du peintre. Le tableau désigné par ses composantes matérielles répond à cette vision métonymique du poète. Ce dernier ajoute un dernier fragment, mi littéral, mi métaphorique, matériel, pourvu d'une fonction mimétique, qui dépasse l'effet de la beauté considérée : le cour vient rendre sensible et matérielle une image évanescente et absente. A rebours, une vision idéaliste de la beauté se défie de cette pensée du fragment et suggère qu'on s'intéresse plus à la manière qu'à la matière.

Ce lieu charnel de représentation, le cœur, surgit ainsi face à une aporie de la représentation. Un certain nombre de pièces évoque l'impossibilité de redoubler le visage de la femme qui est qualifié de «nonpareil ». La peinture ne peut le représenter car il est unique. Théophile pousse à l'extrême cette dissemblance entre l'image et son modèle en retournant contre les arts leur argument majeur. Eux qui sont capables de donner l'immortalité au sujet de leur œuvre, voici qu'ils se heurtent au caractère séculaire de l'ouvrage naturel. Théophile, dans une perspective plus platonicienne, moins soucieux de matérialiser les images que d'assigner à l'art, la restitution d'idées intelligibles, souligne 
la double impossibilité de représenter le beau, qui suppose une invention supérieure à celle de la nature et une temporalité qui échappe au peintre :

Qui voudrait faire une peinture,

Qui put ses traits représenter,

Il faudrait bien mieux inventer

Que ne fera jamais nature.

Tout un siècle les Destinées

Travaillèrent après ses yeux,

Et je crois que pour faire mieux

Le temps n'a point assez d'années ${ }^{29}$.

Si le peintre ne peut copier cette beauté, ni rivaliser avec le travail des Destinées, le poète vient de la dessiner. La limite technique et esthétique du peintre suggère une mimesis impossible, ni sensible, ni intelligible, soumise à la nécessité de l'invention plus que de l'imitation, talent assignable au poète. Cette prétérition alambiquée et cette fausse humilité donnent la victoire à la poésie en présentant un portrait en vers et un miroir naturel écrivant justement la venue d'une Vénus ${ }^{30}$, liant ainsi la fugacité du support et le caractère éphémère des mots qui sont écrits au fil de l'eau. Théophile joue de visions paradoxales de la création artistique : l'œuvre d'art doit dépasser la nature, non parce qu'elle lui est supérieure, ou qu'elle se refuserait à l'imiter, comme on l'attendrait au regard des théories sur l'œuvre d'art, mais pour être capable de la représenter et faire comprendre sa beauté. Ce «mieux faire », cette manière, est du reste au cœur de la poétique de Théophile et passe davantage par le trait du dessin, que par la gravure sculptée, le langage et l'écriture. Une anthologie contemporaine supprime ce passage sur la peinture impossible ${ }^{31}$ se limitant à l'évocation du miroir écrivain et annihilant la présence du peintre. Elle propose une autre pièce qui vient expliciter ce « mieux faire » par un « dire mieux » :

Il faudrait inventer quelque nouveau langage,

Prendre un esprit nouveau, penser et dire mieux

Que n'ont jamais pensé les hommes et les Dieux,

Si je parviens au but où mon dessein m'appelle

Mes vers se moqueront des ouvrages d'Apelle ${ }^{32}$.

L'invention du langage et de la pensée, les deux sens du logos grec, s'inscrit donc contre l'imitation poétique et picturale et surtout l'illusion parfaite dont Apelle est le symbole (puisqu'il trompe même les autres peintres comme Zeuxis) et toute référence à la matière. Après la critique du rimailleur qui

\footnotetext{
${ }^{29}$ Théophile, « La Solitude », Crème des bons vers, p. 274. (éd. Saba, Paris, Garnier, 2008, v.77-84, p.59).

${ }^{30}$ « Présente lui ta face nue/ Tes yeux avec l'eau riront/Et dans ce miroir écriront/Que Vénus est ici venue », Baudoin/Du Bray, p. 293. (éd. Saba, Ibid., v.109-112, p. 63).

${ }^{31}$ La version de «la Solitude» publiée par Baudoin/Du Bray, 1620, p.293, est amputée de trois strophes dont les strophes éditées dans la Crème.

32 «Satyre troisième », Baudoin/Du Bray, 1620, p.332. Cette satire est éditée dans les œuvres complètes sous le titre « Elégie à une Dame » (éd. Saba, Paris, Garnier, 2008, p.119, v.154-158).
} 
n'est capable que de copier Malherbe, Apelle est à son tour dénigré. La conversion dysphorique de la figure d'Appelle est d'autant plus étonnante que Théophile ne s'en prenait pas frontalement à Malherbe dans les vers qui précèdent, mais à ses imitateurs et que dans une élégie, il le recommande comme modèle ${ }^{33}$. Dans les anthologies de Du Bray, on remarque qu'Apelle est reconnu comme le modèle du peintre, de même que Malherbe devient peu à peu le modèle poétique. Théophile en s'en prenant au peintre modèle comme aux élèves de Malherbe, marque sa différence et sa nouveauté qu'ils renvoient dos à dos. La métaphore picturale est alors utilisée pour évoquer une critique de l'illusion et le mensonge de la représentation, de ce qu'on pourrait appeler un « mal faire » :

Lorsqu'il faut cacher cent défauts

Afin que deux vertus éclatent :

Nos esprits d'un pinceau divers

Par l'artifice de nos vers

Font le visage à toutes choses,

Et dans le fard de leurs couleurs

Font passer de mauvaises fleurs

Sous le teint de lys et de roses ${ }^{34}$.

Hésitant entre un enjeu esthétique et éthique, la critique de la peinture passe par la mise en évidence du caractère naturel et vrai du dessin poétique, signe d'un respect de la nature comme d'un refus de la soumission sociale à des autorités qu'il faudrait louer. Les vers sont comme le miroir de l'onde, mais ils sont suffisamment immatériels pour rendre compte du beau. Théophile construit donc une aporie dans la matérialisation du tableau: la dissemblance du tableau et de son modèle semble incontournable au regard de la dimension intelligible et idéale du sujet à représenter, liant au beau le vrai, lors même que le tableau est inscrit dans le leurre et le défaut de perfection.

Cette image idéale de la beauté féminine, soumise à une exigence croissante du naturel, entraîne fort logiquement le poète moins enclin au monde platonicien ou soucieux de proposer une forme réflexive fidèle dans sa matière, à inventer une autre copie sensible de la femme : au miroir naturel, assez attendu, le poète substitue parfois l'image dans son cœur, l'image mentale au lieu de l'image idéale de Théophile. Il ne s'agit plus de comparer l'image picturale et le vrai visage mais la peinture vive de femme naturellement créée et l'image peinte par l'amour «vivement» dans le cœur du

\footnotetext{
33 «Thisbé pour le portrait de Pyrame au peintre », éd. Saba, Paris, Garnier, 2008, v.27, p. 259. Cette pièce est absente des anthologies. On y retrouve néanmoins des traits repérés chez d'autres auteurs : la représentation métonymique du sujet ( « apporte-moi demain/Le portrait de l'œil de Pyrame/Ou celui de sa belle main », v. 8-10), l'assemblage des éléments les plus beaux (« en un mot sais-tu tous les traits/de toutes les plus belles choses »?, v. 23-25); la relation entre la nature réflexive et la peinture (« Tu peindrais Pyrame[...]/ou ce premier éclat du jour/ Lorsque [...]/Dans le ciel comme un tableau/il fait luire son visage/tout fraîchement sorti de l'eau », v.45-50).

${ }^{34}$ Théophile, « Ode à Montmorency », Baudoin/Du Bray, 1620, p.344.
} 
poète. Cette comparaison inscrit le cœur dans la cohorte des supports réflexifs. Elle est l'occasion de marquer une nouvelle concurrence entre original et copie :

Si l'un des yeux fermé, de l'autre seulement

Je regarde attentif votre divin visage,

Ma belle, pour cela n'entrez point en ombrage,

Je n'ai de ma blessure aucun ressentiment.

Mais Amour en mon cœur ayant peint vivement

Avec un de ses traits, votre céleste image,

Il veut que comparant au patron son ouvrage

D'un si parfait rapport je fisse jugement.

Doncques en même temps tournant une prunelle

Vers le portrait du cœur, l'autre vers le modèle,

Je vous contemple en vous, et vous contemple en moi

Mais l'art va de si près imitant la nature

Que je doute laquelle est la peinture

Et lequel de mes yeux mérite le plus de foi ${ }^{35}$.

Le dédoublement du regard se traduit par un dédoublement de deux images désignées par le dernier vers comme aussi vraies ou trompeuses l'une que l'autre. Le premier quatrain est plus ambigu, car il s'interroge sur la réaction sensible de l'amant face au vrai visage, réaction atténuée (« aucun ressentiment») au regard de l'image du cœur peinte «vivement», qui suggère l'animation de l'image et sa sensibilité. L'imitation du cœur est ainsi plus sensible et le modèle non moins illusoire que cette copie. La peinture du cœur partage ainsi ses attributs avec la beauté réelle. Ailleurs on lui assigne la durée qui manque au modèle et au tableau peint: Baro a perdu la femme qu'il aime, le « beau portrait dont [son cœur] adorait le trait» mais

[Il sait] bien que jamais malheur

N'ôtera l'autre que je porte

Gravé si vivement au milieu de [son] cœur $^{36}$.

La peinture du cœur est la meilleure : elle a le même effet que le modèle ou le tableau, reste animée et vivante. Elle a l'ubiquité et la pérennité que n'a pas la peinture naturelle ni artificielle, soumise à une matérialité extérieure au corps du poète. On voit donc bien là encore que la temporalité est au cœur de la réflexion sur la copie. Si Théophile voyait dans le caractère séculaire de la beauté le signe de l'impossibilité de sa reproduction par l'être humain, l'image mentale rend durable l'évanescence de la belle absente. Ce décentrement de la question de la belle image idéale vers l'invention d'une image mentale, vraie et pérenne semble justement emprunté à la matérialité de la peinture : un dessin

\footnotetext{
${ }^{35}$ Meziriac, « Sur une blessure en un œil », Baudoin/Du Bray, 1620, p. 574.

${ }^{36}$ Baro, « Sur la perte d'un portrait », Baudoin/Du Bray, 1620, p. 362.
} 
d'Alcidon ${ }^{37}$ le présente tenant dans ses bras un portrait de son amour, Daphnide ravie par Jupiter, et lui fait dire que « depuis jour et nuit [il] la tient dans [ses bras] ». Cette fétichisation du tableau est reprise aussi par Boisrobert qui se réjouit de pouvoir tenir « toujours embrassé " ${ }^{38}$ le tableau de la femme qu'il aime. Pour reprendre une distinction platonicienne opposant la copie et le simulacre et l'icône et l'idole, l'amant a à choisir entre l'idolâtrie du tableau qui vient se substituer à la femme aimée et apparaît différent d'elle, simulacre fait de matières différentes, la copie du cœur qui conserve et possède la sensibilité et l'intelligibilité de la beauté aimée, l'idée seule de la beauté. C'est justement l'enjeu d'une pièce de Boisrobert qui évoque d'un tableau les « faux appas/qu'[il] connaît dans une idole » auquel « rien de bon ne [lui] en succède ${ }^{39}$. Son désir est certes satisfait, mais il ne change rien à l'histoire d'amour que lui refuse toujours sa belle et à son incapacité à saisir sa beauté.

\section{- Le peintre, figure mythologique.}

Cette distinction permet de comprendre un dernier usage de la peinture : en marge des images mentales et idéales, comme simulacre, elle participe au final à la création de la fiction fantasmée où il ne s'agit plus ni de vérité ni de perfection. Au-delà des supports réflexifs avec lesquels elle rentre en concurrence et avec lesquels elle échange ses attributs, jusqu'à vouloir se substituer au modèle reproduit, elle est le lieu de réinvention de mythes, syncrétiques, fait d'assemblages à l'instar des portraits. Le peintre s'y voit convoqué par le poète et transformé en figure héroïque. Plusieurs pièces témoignent de cette imbrication entre l'image idolâtrée et le réseau syncrétique mythologique renouvelé.

Le tableau décrit par Racan, où l'on voit Alcidon et Daphnide, est assez exemplaire du travail du poète se mêlant de donner au tableau une portée fantasmatique dans un jeu syncrétique de mise en abyme des mythes. Racan croise l'univers mythologique avec le romanesque de l'Astrée. Il ajoute à la nouvelle de d'Urfé, un élément nouveau et inventé (l'enlèvement par Jupiter) quoique récurrent dans la mythologie (Ganymède ou Europe sont enlevés par Jupiter). L'étreinte permanente des amants tous deux peints, mais à deux niveaux différents (Alcidon peint tient le portrait de Daphnide) est elle-même empruntée à deux sources, l'une évidente, Ovide dans ses Métamorphoses passe son temps à réunir des couples pour l'éternité sous des formes matérielles (végétaux, étoiles), l'autre directement liée aux vers évoquant justement la peinture dans les anthologies, tel Ixion. Il est certes trompé par Jupiter fabriquant un nuage à la semblance d'Héra, son épouse désirée par Ixion, mais il possède néanmoins physiquement ce nuage. Boisrobert, édité dans le même recueil que Racan, dit

\footnotetext{
${ }^{37}$ Racan, « Inscription pour mettre dessous un tableau où Alcidon est peint, tenant Daphnide entre ses bras », Du Bray, 1627, p. 232. Il reprend un épisode des amours de Daphnide et d'Alcidon (H. d'Urfé, l'Astrée, tome III, livres III et IV).

${ }^{38}$ Boisrobert, «Sur un portrait », Du Bray, 1627, p. 482.

${ }^{39}$ Ibid., p. 484.
} 
qu'il « contenta son désir $»^{40}$. Cette satisfaction, même éphémère et coûteuse, est sensible. Comme lui, Alcidon possède une chimère, vaine image de Daphnide et s'en contente un temps. Jupiter est convoqué, mais comme ravisseur illégitime de Daphnide, dans un jeu d'inversion en miroir du mythe d'Ixion. Ainsi l'amant torturé sur une roue chez les poètes de la fin du XVI ${ }^{\mathrm{e}}$ siècle, change de visage, lorsqu'intervient l'art pictural : représentant le héros au début de son histoire, l'effet produit par l'illusion compte davantage que la punition qu'il subira. L'imagination et le succès de ses sensations transforment la portée morale du mythe qui insiste plus sur la perfection sensible de l'imitation que sur le danger physique et moral du désir. L'idole est bien reconnue comme idole mais elle offre cependant à l'amant, un plaisir que refusait le récit mythologique. Même constat quand le peintre, à la semblance d'Actéon reste encore impuni chez Maynard:

Ton œil trop hardiment prompt

A vu cette déité nue $e^{41}$

Le héros supplicié présenté par les poètes baroques contemporains, dans ses tourments, est plutôt utilisé comme un avertissement euphémisé donné au peintre : au lieu de nous représenter l'amant, le portrait de Diane montre la culpabilité du peintre, devient un motif du mythe qui pourra justifier la vengeance de la déesse. La peinture est inscrite dans un double réseau imaginaire : le portrait imaginaire présenté par les vers en ekphrasis qui lui confère une réalité descriptive, le mythe qui se voit ici offrir une nouvelle variante, au moment même où Théophile publie son « épitre d'Actéon à Diane ». Dans cette épitre, le chasseur écrit à Diane pour lui décrire la manière dont il l'a observée. La lettre devient la cause de la mort d'Actéon, démasqué, mais cette mort n'est que suggérée en toute fin de lettre, comme ici. Le tableau ici remplace la lettre mais procède des mêmes procédés : faire surgir au cœur du mythe comme motif signifiant et décisif, une œuvre artistique, euphémiser la mort violente sur laquelle s'attardaient les poètes de la génération précédente, motiver par un élément de la narration, la métamorphose. Vermeil, qui relève encore de celle-ci, atténuait déjà le destin du peintre en l'avertissant contre les dangers qui le guettent, plutôt qu'en le représentant torturé :

Garde-toi d'imiter le foudre de ses yeux,

De peur d'être puni comme fut Salmonée.

Peins sa bouche en bouchant ton oreille étonnée $[\ldots]$

Mais lorsque tu auras parfait tout le tableau

Garde-toi d'être fait toi-même ton tombeau,

Empierré sans merci de son regard habile :

\footnotetext{
${ }^{40}$ Boisrobert, « Sur un portrait », Du Bray, 1627, p. 483.

${ }^{41}$ Maynard, « Pour un Peintre qui s'était acquis de la réputation par un portrait de Diane », Conti/Du Bray, 1620, p. 1045 et Du Bray, 1627, p. 375.
} 
Mon penser le peignit jadis dedans mon cœur

Et reçut pour loyer le rocher son vainqueur ${ }^{42}$.

Contrairement à Salmonée puni d'avoir imité Zeus, et au poète médusé, lié au destin d'une pierre comme Prométhée ou Sisyphe, à l'instar d'Ulysse échappant aux sirènes et à Méduse, le peintre profite de la prudence du poète qui lui propose une contre-manière («garde-toi ») pour faire son tableau. Vermeil inverse la forme de l'art poétique pour proposer un art pictural inversé : on se démarque de l'histoire de Timanthe, le peintre qui pour dire la douleur, voilait le visage d'un homme ; on reprend aussi l'idée que le tableau produit des effets similaires à son modèle tout en suggérant que le peintre peut seul échapper aux effets de son œuvre. C'est au peintre de voiler et de maîtriser ses sens. Ces références aux mythes désignent donc un paradoxe du peintre : il doit renoncer à sa perception pour reproduire le monde, et se détacher de son univers personnel (Timanthe) pour dépasser tous les héros et le poète lui-même. Prométhée, choisi à son tour comme avatar mythologique du peintre, souligne toute la complexité de la mythologisation de l'univers pictural : il vole le feu aux dieux pour le donner à l'homme, et leur offre ainsi le langage. Or, la peinture est chose muette. Pour conjurer cette invraisemblance, le poète décide que le feu de Prométhée est identifiable au souffle créateur de l'âme et présent dans l'éclat des yeux : le peintre pour saisir cette lumière du regard a volé le feu :

Peut-il vous avoir imitée

Jusqu'à l'éclat de vos yeux

Sans dérober le feu des cieux

Comme fit jadis Prométhée ${ }^{43}$.

Héros mythologique, le peintre n'est plus inspiré par les dieux, il a dérobé leur bien. Le poète à son tour, pour donner vie et corps au tableau qu'il contemple, ne peut que souhaiter « pouvoir ravir le feu des cieux $»^{44}$. Ailleurs on trouve une correspondance entre la matière utilisée pour le tableau et la figure du peintre : la cire de ses couleurs motive l'apparition d'Icare

Icare nous devait apprendre

De ne voler pas dans les cieux.

Desseignant contre la coutume

Ce qu'un mortel ne devait pas :

Le Soleil, la cire et la plume

Furent cause de son trépas.

\footnotetext{
${ }^{42}$ Vermeil, «Sonnet », Seconde partie des Muses françaises ralliées de diverses parts, Guillemot, 1600, p. 218 . (éd. Lafay, Genève, Droz, VII, v.3-14, p. 43.

${ }^{43}$ Boirsobert, « A Madame de Chevreuse, sur son portrait », Du Bray, 1627, p.448.

${ }^{44}$ Boisrobert, « Sur un portrait », Du Bray, 1627, p. 484.
} 
Toi pour peindre, moi pour écrire,

Nous courons un semblable sort :

Ces soleils, ma plume et ta cire

Seront cause de notre mort

Mais achève sa belle face

Seul tu n'auras pas la douleur :

Le compagnon de ton audace

Sera celui de ton malheur ${ }^{45}$.

A l'inverse des autres vers, le poète pousse plus clairement l'autre artiste à la rivalité avec les dieux et l'entraîne dans une douleur mortelle. Il conserve la cohérence élémentaire entre la cire des couleurs vue plus haut et celle des ailes d'Icare, qui motive l'apparition de la figure mythologique. La matérialité du tableau porte la logique du texte. Le poète doit changer d'outil et change son miel contre une plume. Il passe de la matière à la manière, laissant au peintre son élément, et résout ainsi la dichotomie qu'on avait perçue plus haut entre ces deux pôles. Le partage des deux artistes de la même mort est surprenant : au lieu de l'union amoureuse avec la beauté peinte ou la satisfaction de voir son désir un temps satisfait par son portrait à disposition, ce sont les deux artistes qui se rejoignent dans la mort, de même que leur création partait d'une matière commune déclinée en deux natures, cire et miel de l'abeille. Cette communion des arts résout pour un temps la comparaison entre leurs œuvres et suggère une harmonie des techniques au cœur du chaos amoureux.

Notons pour terminer sur la mythologisation de la peinture que toutes ces pièces ne sont pas des fables $^{46}$ et ne convoquent pas les héros sous forme de mythologismes. Ce sont davantage des présentations de tableaux imaginaires ou encore virtuels qui ne se posent plus comme reflet de la nature, mais visions déformées de figures : l'imitation est ainsi rejetée, puisque on s'éloigne de la perspective de la nature comme celle des auteurs à copier. La poésie réécrit le mythe, invente peinture et héros. Elle inscrit la peinture dans l'intrigue : le dessin est chargé de jouer un rôle dans le nœud de l'histoire, de même que le peintre, devenu acteur à la semblance mythologique. Le tableau reflète le nouveau mythe à trois niveaux : il dessine son contenu, sa création dépend de l'épreuve imposée à son auteur, sa présence est décisive pour la fin du mythe.

Ainsi la poésie dans un rapport à la peinture est inscrite dans deux mouvements paradoxaux : à la matérialisation complexe du tableau répond la fictionnalisation du peintre devenu héros mythologique, paradoxe qui rend compte de la dualité de la peinture, objet tangible et sujet feint et

\footnotetext{
${ }^{45}$ Porchères, « Stances sur un portait de cire », Le Séjour des Muses ou la crème des bons vers, Rouen, Daré, 1626, p. 381-382.

${ }^{46}$ Contrairement à ce qui se passe à la Renaissance. Sur la fable et le tableau, voir Olivia Rosenthal, Donner à voir : écritures de l'image dans l'art de poésie au XVI siècle, Paris, Champion, 1998, p. 349-374.
} 
de la volonté de dépasser dans les vers la question de sa vérité. Elle montre en effet toute la complexité de la notion d'imitation : le tableau vient la partager avec des supports réflexifs qui lui font concurrence (le miroir ou le cœur) tout en lui conférant ou en lui empruntant des attributs essentiels : animation, beauté, sensibilité, ubiquité et pérennité de l'image produite. L'effet du tableau, à l'aune du sujet qu'il reproduit, est au final plus prégnant que la fidélité visuelle avec le modèle dont il s'inspire. La fonction de renouvellement rhétorique du cliché poétique opéré grâce à la mention de la peinture va de pair avec la modification des mythes qui se croisent pour donner au peintre les traits d'un héros paradoxal, auteur de tableaux sensibles qu'il doit peindre en renonçant à ses sensations propres. La poésie utilise donc le motif pictural pour décentrer son monde et échapper à une esthétique qui devient pesante : au lieu du miroir et de l'illusion, du héros supplicié et de la multiplicité disloquée empruntés aux baroques, elle invente d'autres traits pour ces lieux et figures qui lui confèrent toujours une place centrale de témoin et de juge et auxquels elle tente à tout prix de donner une unité sensible, liant soigneusement des fragments du beau à défaut d'être idéale, et une vérité constante. En marge de sa représentation de la peinture, la poésie définit progressivement de nouvelles frontières qui se soucient beaucoup moins d'éthique que d'esthétique et tentent d'étager la fiction en plusieurs univers parallèles. Il ne s'agit plus de copier le réel, mais de décrire, de cerner et d'inventer toutes les modalités possibles de ce réel dont le tableau peint serait la synthèse parfaite à la fois objet tangible, copie sensible, simulacre assouvissant le désir, mythe. Nous nous retrouvons alors face à un réseau d'images étageant poésie et peinture en marge du réel. Laissant peu à peu la simple pensée analogique, la poésie des recueils convoque pour évaluer la peinture une taxinomie nuancée et complexe de l'image dans l'imitation qu'elle lui associe ou au contraire dont elle lui interdit l'accès : figure sensible, tangible ou virtuelle, elle se distingue des représentations mentale ou idéale. Modernité du recueil collectif devenu encyclopédie d'images, et non plus seulement univers analogique.

\section{Liste des recueils collectifs de pièces en vers généralistes du début du XVII siècle}

\section{Editions de Raphaël et Daniel du Petit Val}

Recueil de Diverses poésies, tant du feu sieur de Sponde, que des sieurs du Perron, de Bertaud, de Porchères, et autres non encore imprimées, Rouen, Raphaël du Petit Val, 1597.

Diverses poésies nouvelles données à R.D.P.Val par ses amis, Rouen, Raphaël du Petit Val, 1597.

Second recueil de diverses poésies des plus excellents auteurs de ce temps, Rouen, Raphaël du Petit Val, 1599.

Troisième recueil des plus excellents auteurs de ce temps, 1600.

Quatrième recueil des plus excellents auteurs de ce temps, 1600.

Premier Recueil de divers poésies tant du feu sieur Sponde que du sieur Du Perron, de Bertaud, de Porchères et autres non encore imprimées, Rouen, Raphaël du petit val, 1604 [réed de 1597]. 
Diverses poésies nouvelles données à R.D.P.Val par ses amis, Rouen, Raphaël du Petit Val, 1606 [réed de 1597]

Le Temple d'Apollon ou nouveau recueil des plus excellents vers de ce temps, Rouen, Raphaël du Petit Val,1611,[2 t.

Le Cabinet des Muses ou nouveau recueil des plus beaux vers de ce temps, Rouen, Daniel du Petit Val, 1619.

\section{Editions de Mathieu Guillemot}

Les Muses ralliées de diverses parts ou le Parnasse des plus excellents poètes de ce temps, éd. Despinelle, Paris, Guillemot, 1598, 1599, 1603 puis Lyon, Ancelin 1606, 1609, 1618.

Seconde partie des Muses ralliées de diverses parts, Paris, Guillemot, 1600.

Le Parnasse des poètes français, éd. Gilles Corrozet, Guillemot, 1607.

Le Nouveau Parnasse, Paris, Guillemot, 1609 [copie de Du Bray, 1609.

Le Parnasse des plus excellents poètes de ce temps, Guillemot, 1618 [éd de 1607].

\section{Editions de Toussaint du Bray}

Nouveau Recueil des plus beaux vers de ce temps, éd. de Malherbe ( ?), Paris, T. du Bray, 1609.

Nouveau recueil de Délices de la poésie française, éd de Fr de Rosset, Paris, Du Bray, 1615 et 1618. [Les Muses en deuil, Paris, Toussaint Du Bray, 1620 : éd de circonstance].

Second livre des Délices de la poésie française ou nouveau recueil des plus beaux vers de ce temps, éd. Jean Baudoin, Paris, Du Bray, 1620.

Les Délices de la poésie française ou dernier recueil des plus beaux vers de ce temps corrigé de nouveaux par les auteurs et augmenté d'une élite de plusieurs rares pièces non encore imprimées, déd. à Princesse de Conti, Paris, Du Bray, 1620 [rééd. Partielle de l'éd de 1618 et 1620] Apollon, Paris, Du Bray, 1623, [version abrégée des Recueils des délices].

Recueil des plus beaux vers de Messieurs de Malherbe, Racan, Monfuron, Maynard, Boisrobert, l'Estoille, Lingendes, Touvant, Motin, Mareschal, et autres des plus fameux esprits de la cour, Paris, Du Bray, 1626.

\section{Editeurs divers}

Recueil des plus diverses poésies tant de M. du Perron que des sieurs Bertaud, Porchères, et d'autres, Paris, Bonfons, 1598.

Les Fleurs des plus excellents poètes, Paris, Bonfons, 1599 et 1601.

L'Académie des modernes poètes remplies des plus beaux vers que ce siècle réserve à la postérité, éd. par Nervèze Paris, Du Breuil, 1599.

Marguerites poétiques tirées des plus fameux poètes français, tant anciens que modernes, réduites en forme de lieux communs et selon l'ordre alphabétique, nouvellement recueillies et mise en lumière par Esprit Aubert, Lyon, Barthélémy Ancelin, 1613.

Le Séjour des Muses ou cresme des bons vers de De Lingendes. Ronsard, Bertaut, Théophile etc., Rouen, Daré 1622. 